\title{
Mig-2 attenuates cisplatin-induced apoptosis of human glioma cells in vitro through AKT/JNK and AKT/p38 signaling pathways
}

\author{
Yun-wei OU ${ }^{1,2}$, Zi-tong ZHAO ${ }^{2}$, Chuan-yue $\mathrm{WU}^{3}$, Bai-nan $\mathrm{XU}^{1, *}$, Yong-mei SONG ${ }^{2, *}$, Qi-min ZHAN ${ }^{2}$ \\ ${ }^{1}$ Department of Neurosurgery, Chinese PLA General Hospital, Beijing 100853, China; ${ }^{2}$ State Key Laboratory of Molecular Oncology, \\ Cancer Institute and Cancer Hospital, Chinese Academy of Medical Sciences and Peking Union Medical College, Beijing 100021, \\ China; ${ }^{3}$ Department of Pathology, University of Pittsburgh, Pittsburgh, PA 15261, USA
}

\begin{abstract}
Aim: Mig-2 (also known as Kindlin-2 and FERMT2) is an important regulator of integrin activation and cell-extracellular matrix adhesion, and involved in carcinogenesis and tumor progression. The aim of this study was to investigate the role of mig-2 in cisplatin-induced apoptosis of human glioma cells in vitro.

Methods: The expression of mig-2 was modulated in human glioma H4, HS 683 and U-87 MG cells by transfection with a plasmid carrying mig-2 or mig-2 siRNA. Cisplatin-induced apoptosis was detected using Annexin V/PI staining and flow cytometry, as well as MTS analyses. The expression of apoptosis-related or signaling proteins was examined using Western blotting analysis. H4 cells were transfected with plasmids carrying mig-2 mutants to determine the functional domain of mig- 2 .

Results: In the 3 glioma cell lines tested, overexpression of mig-2 significantly attenuated cisplatin-induced apoptosis, whereas knockdown of mig-2 potentiated the apoptosis. The mechanisms of action of mig- 2 were further addressed in $\mathrm{H} 4$ cells: overexpression of mig-2 markedly reduced cleaved caspase-9, caspase-8, caspase-3 and PARP, as well as p-JNK and p-p38, and increased p-AKT in cisplatin-treated $\mathrm{H} 4$ cells, whereas mig-2 siRNA reversely changed these apoptosis-related and signaling proteins. Furthermore, pretreatment with JNK inhibitor SP600125 and p38 inhibitor SB203580, or with AKT inhibitor LY294002 abolished the effects of mig-2 on cisplaxtin-induced apoptosis. In H4 cells, GFP-mig-2 F3 plasmid that contained only the F3 subdomain showed the same efficiency in attenuating cisplatin-induced apoptosis, as the mig-2 wild-type vector did, whereas GFP-mig-2 (1-541) plasmid that lacked the F3 subdomain was inactive.

Conclusion: Mig-2 significantly attenuates the antitumor action of cisplatin against human glioma cells in vitro through AKT/JNK and AKT/p38 signaling pathways. The F3 subdomain of mig-2 is necessary and sufficient for this effect.
\end{abstract}

Keywords: glioma; mig-2; cisplatin; apoptosis; caspases; AKT; JNK; p38; siRNA

Acta Pharmacologica Sinica (2014) 35: 1199-1206; doi: 10.1038/aps.2014.60; published online 25 Aug 2014

\section{Introduction}

Human gliomas are the most common primary tumors in the central nervous system, accounting for $70 \%$ of human primary malignant brain tumors ${ }^{[1]}$. The current treatment strategy for malignant glioma is surgery combined with radiotherapy and chemotherapy. However, due to chemotherapy and radiotherapy resistance and our poor understanding of the molecular mechanisms of glioma, the overall prognosis for patients with malignant glioma is not optimistic. In patients diagnosed with intermediate-grade malignant glioma or glioblastoma, the

\footnotetext{
* To whom correspondence should be addressed.

E-mail symlh2006@163.com (Yong-mei SONG); xbn301sjwk@126.com (Bai-nan XU)

Received 2014-03-30 Accepted 2014-05-26
}

median survival is 3-5 years or 19-23 months, respectively ${ }^{[2]}$. Therefore, it is crucial to understand the pivotal molecular mechanisms of and identify new and effective therapeutic targets for glioma.

As a member of the kindlin family, mig-2 (also known as Kindlin-2 and FERMT2) is characterized by a FERM domain, which consists of F1, F2, and F3 subdomains. The F2 subdomain is bisected by a pleckstrin homology $(\mathrm{PH})$ domain $^{[3]}$. Functionally, mig-2 is a significant regulator of integrin activation and cell-extracellular matrix (ECM) adhesion ${ }^{[4]}$, and it is related to carcinogenesis and tumor progression in breast cancer, gastric cancer and prostate cancer ${ }^{[5-7]}$. However, the function of mig-2 in regulating glioma tumorigenesis has not been reported.

In this study, we investigated the influence of mig-2 in 
the mediation of glioma cell apoptosis and the underlying mechanisms of this influence. We determined that mig-2 played an antagonistic role in cisplatin-induced apoptosis in glioma cells, and this process was involved in the activation of caspase-8, caspase-9, and caspase-3. We also found that mig-2 antagonized cisplatin-induced apoptosis through AKT/ JNK and AKT/p38 signaling pathways. Furthermore, the F3 subdomain of mig-2 was necessary and sufficient for mig-2 antagonism of cisplatin-induced apoptosis. In summary, our results suggest that mig-2 may be an ideal marker for screening glioma patients for cisplatin therapy and that mig-2 could also be a potential therapeutic target in glioma.

\section{Materials and methods Cell culture}

The human glioma cell lines H4, HS 683, and U-87 MG were purchased from the Cell Culture Center (Chinese Academy of Medical Sciences, Beijing, China) in June 2011. As previously described $^{[8]}$, H4 and HS 683 cells were grown in Dulbecco's modified Eagle's medium supplemented with $10 \%$ fetal bovine serum. U-87 MG cells were cultured in minimum essential medium supplemented with $10 \%$ fetal bovine serum. The cells were maintained under standard cell culture conditions at $37^{\circ} \mathrm{C}$ and $5 \% \mathrm{CO}_{2}$ in a humid environment.

\section{Antibodies and special reagents}

Cleaved caspase- 3 antibody, cleaved caspase- 8 antibody, cleaved caspase- 9 antibody, cleaved PARP antibody, Bid antibody, Bax antibody, Bcl-2 antibody, cytochrome $c$ antibody, AKT antibody, p-Akt (Ser473) antibody, ERK5 antibody, p-ERK5 (Thr218/Tyr220) antibody, ERK1/2 antibody, p-ERK1/2 (Thr202/Tyr204) antibody, JNK antibody, p-JNK antibody (Thr183/Tyr185), p38 antibody, p-p38 (Thr180/ Tyr182) antibody, GFP antibody and $\beta$-actin antibody were purchased from Cell Signaling Technology, Inc (Danvers, MA, USA). The anti-mig-2 antibody was a kind gift from Dr Chuan-yue WU (University of Pittsburgh, USA). Horseradish peroxidase-conjugated anti-rabbit or anti-mouse antibody, caspase-3 inhibitor Z-DEVD-FMK, caspase-8 inhibitor Z-IETDFMK, caspase-9 inhibitor Z-LEHD-FMK, AKT inhibitor LY294002, JNK inhibitor SP600125, and p38 inhibitor SB203580 were purchased from Sigma, Inc (St Louis, MO, USA).

\section{Oligonucleotide transfection}

All mig-2 plasmids were a kind gift from Dr Chuan-yue WU. Specific siRNA targeting mig-2 was custom-designed and provided by Ribobio (Guangzhou, China). Cells were seeded at $10 \times 10^{4}$ per well in $60-\mathrm{mm}$ culture plates and transfected with oligonucleotides using Lipofectamine 2000 (Invitrogen, Carlsbad, CA, USA) according to the manufacturer's instructions. Transfected cells were incubated at $37^{\circ} \mathrm{C}$ with $5 \% \mathrm{CO}_{2}$ for $48 \mathrm{~h}$.

\section{Western blotting}

Western blotting analysis was performed as follows. The cells were harvested and lysed on ice. The concentration of cellular whole protein was quantified by a colorimetric assay. Eighty micrograms of whole proteins were separated by $10 \%$ SDSPAGE and then transferred to PVDF membranes. After blocking with $2 \%$ bovine serum albumin, the membrane was incubated with primary antibodies overnight. Secondary antibodies conjugated with horseradish peroxidase (HRP) were used to probe the membrane for $1 \mathrm{~h}$. The membrane was rinsed in $1 \times$ PBS with $0.1 \%$ Tween. After incubation with the chemiluminescence substrate, photographs were taken using an Image Reader LAS-4000 (Fuji Ltd, Tokyo, Japan) and analyzed by the Multi Gauge V3.2 software.

\section{MTS}

Cells were seeded into 96-well culture plates at $5 \times 10^{3}$ cells per well, incubated overnight, and transfected with plasmids or siRNAs. Twenty-four hours post-transfection, cisplatin was supplemented in the medium at a suitable concentration, and the cells were maintained for an additional $24 \mathrm{~h}$. Twenty microliters of MTS (CellTiter 96 aqueous one solution reagent, Promega, Madison, WI, USA) diluted in $100 \mu \mathrm{L}$ of culture medium was added to each well of the 96-well culture plates. Cells were incubated for $2 \mathrm{~h}$, and then the absorbance of each well at $490 \mathrm{~nm}$ was recorded using a 96-well plate reader (BioRad Inc, Hercules, CA, USA).

\section{Flow cytometric analysis}

The vital, apoptotic and damaged cells were separated by flow cytometry. The percentage of cells undergoing apoptosis was determined using an Annexin $\mathrm{V}$ apoptosis detection kit (Abcam, Cambridge, UK) according to the manufacturer's instructions.

\section{Mitochondrial membrane potential assay}

Mitochondrial membrane potential $(\Delta \psi \mathrm{m})$ was undertaken using a commercial kit from Beyotime (Beijing, China) according to the manufacturer's instructions. In brief, cells were harvested and labeled with JC- 1 at $37^{\circ} \mathrm{C}$ for $20 \mathrm{~min}$. Relative fluorescence intensities were monitored by flow cytometry. For the detection of JC-1, excitation and emission wavelengths were set at $530 \mathrm{~nm}$ and $580 \mathrm{~nm}$, respectively.

\section{Statistical analysis}

All experiments were performed and repeated at least three times. The data were shown as the mean value $\pm S D$ and were statistically analyzed using a two-sided Student's $t$-test using SPSS 11.5 software. A value of $P<0.05$ was considered statistically significant.

\section{Results}

Mig-2 antagonizes cisplatin-induced apoptosis in glioma cells We first investigated the expression of mig-2 in the human glioma cell lines HS 683, H4, and U-87 MG using Western blotting. Mig-2 was expressed in the three glioma cell lines, and the protein levels of mig- 2 were not significantly different (Figure 1A). Therefore, we transiently transfected HS 683, H4, and U-87 MG cells with flag-mig-2 plasmid, control plasmid, 
A

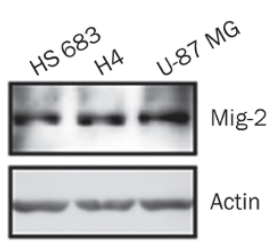

B

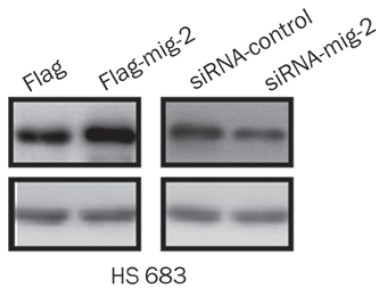

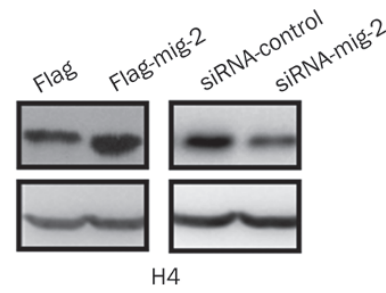

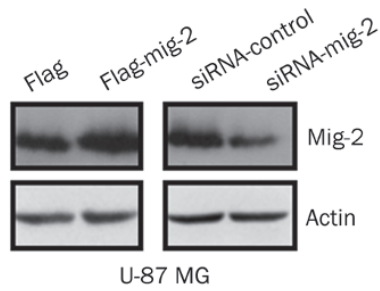

C

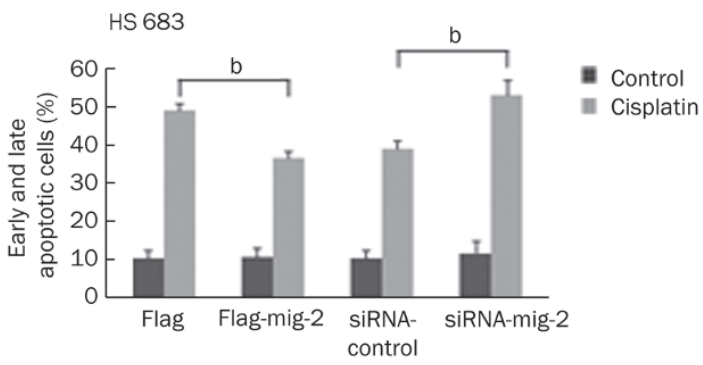

$\mathrm{H} 4$

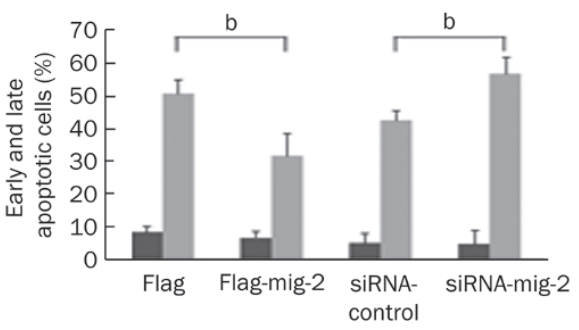

U-87 MG

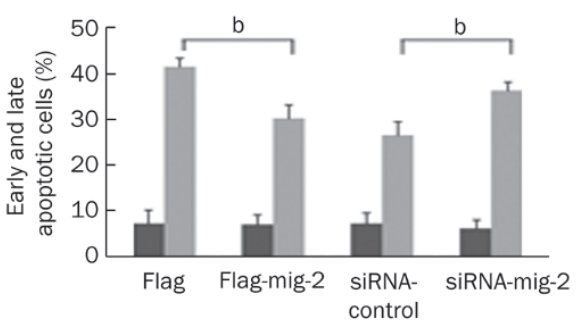

D
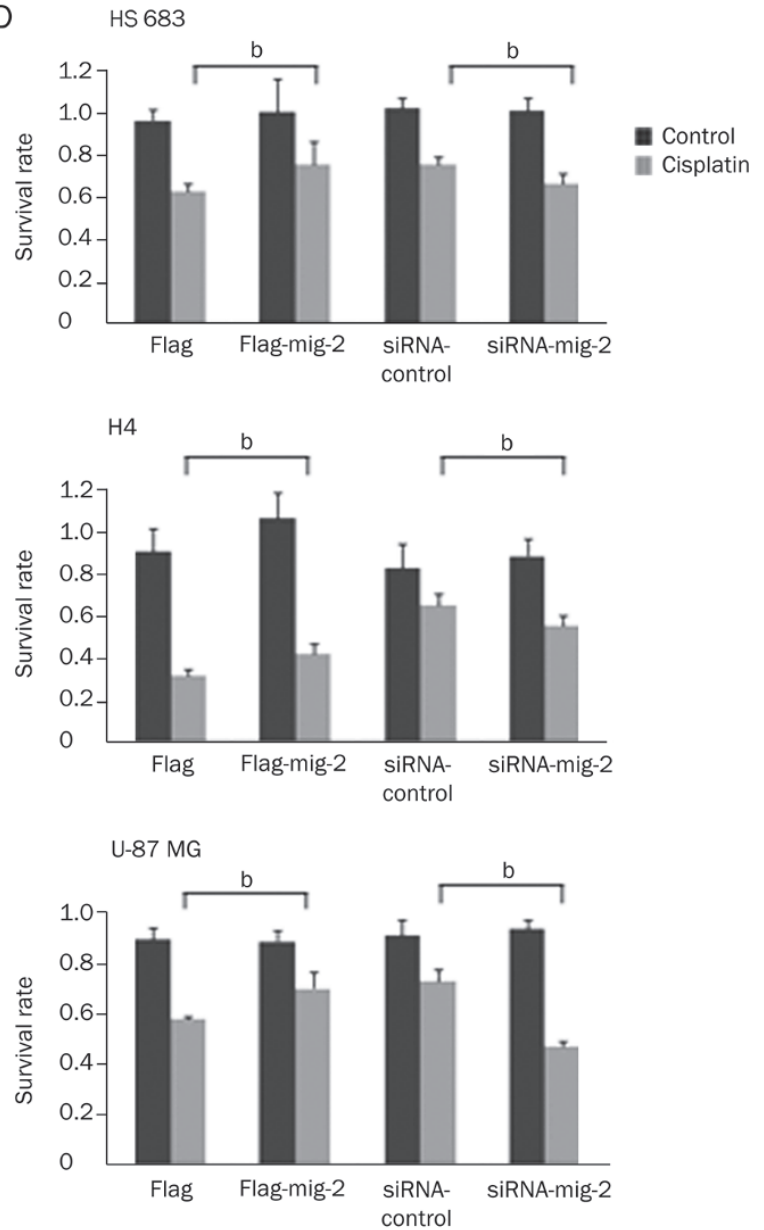

Figure 1. Mig-2 regulated cisplatin-induced apoptosis in glioma cells. (A) Endogenous expression of mig-2 in glioma cell lines HS 683 , H4, and U-87 MG. (B) Ectopic expression or knock-down of mig-2 in glioma cell lines HS 683, H4, and U-87 MG were analyzed using Western blotting using an antimig-2 antibody. (C) Cells were stained using Annexin V/PI for analyzing cell apoptosis. Ectopic expression or knock-down of mig-2 in glioma cell lines. HS 683, H4, and U-87 MG cells were not treated or treated with cisplatin for $24 \mathrm{~h}$. Then, the cells were stained with Annexin V/PI and analyzed by flow cytometry. The bar chart shows the percentage of apoptotic cells. (D) Effects of mig-2 on the viability of glioma cell lines HS $683, \mathrm{H} 4$, and U-87 MG after treatment with cisplatin or a control reagent by MTS assay. The data are presented as the mean \pm SD from three independent experiments. ${ }^{\mathrm{b}} \mathrm{P}<0.05$ versus control.

mig-2 siRNA, and control siRNA. The expression of mig-2 was confirmed using Western blotting (Figure 1B). Then, the cells were treated with cisplatin at 35,50 , and $40 \mu \mathrm{mol} / \mathrm{L}$ for $24 \mathrm{~h}$. The treatment was associated with $50 \%$ apoptotic cells in HS 683, H4, and U-87 MG cells ${ }^{[9]}$. To evaluate the effect of mig-2 on cisplatin-induced apoptosis, Annexin V/propidium iodide (PI) and MTS analyses were performed. After the cells were stained with Annexin V/PI, significant decreases of cisplatin-induced apoptotic cells (early and late apoptosis) in HS
683, H4, and U-87 MG cultures were observed by flow cytometry analysis when mig-2 levels were elevated. By contrast, after mig-2 knock-down through siRNA in HS 683, H4, and U-87 MG cells, cisplatin-induced apoptotic cells were markedly increased (Figure 1C). Similar results were observed in the MTS analysis. As shown in Figure 1D, mig-2 overexpression protected HS 683, H4, and U-87 MG cells from cisplatininduced apoptosis compared with control vectors. However, after cisplatin treatment, the survival rates were significantly 
attenuated when the protein levels of mig- 2 were reduced in HS 683, H4, and U-87 MG cells.

Caspase-9, caspase-8, and caspase-3 activation are involved in mig-2-antagonizing cisplatin-induced apoptosis

To delineate the mechanisms underlying mig-2-mediated apoptosis in glioma cells, we investigated the effect of mig-2 on the activation of caspase- 9 , caspase- 8 , and caspase- 3 and the cleavage of PARP during cisplatin-induced apoptosis in $\mathrm{H} 4$ cells. As shown in Figure 2A, in the control vector group, cells exposed to cisplatin for $24 \mathrm{~h}$ revealed an increased activation of caspase-9, caspase-8, and caspase- 3 and the cleav- age form of PARP compared with the mig-2 overexpression group. At the same time, when exposed to cisplatin, the cleavage of caspase- 9 , caspase- 8 , caspase-3, and PARP was significantly up-regulated in the mig-2 siRNA group compared with the control siRNA-transfected cells in the $\mathrm{H} 4$ cell line. Additionally, during the cisplatin-induced apoptotic process, pro-apoptotic proteins (Bid and Bax) and cytochrome $c$ were reduced, and an anti-apoptotic protein (Bcl-2) was increased in ectopic expression in mig-2 overexpression cells compared with the vector control-transfected cells. The ratio of Bax/ Bcl-2 was also notably decreased in mig-2 overexpression cells. Simultaneously, when treated with cisplatin, silencing endog-
A

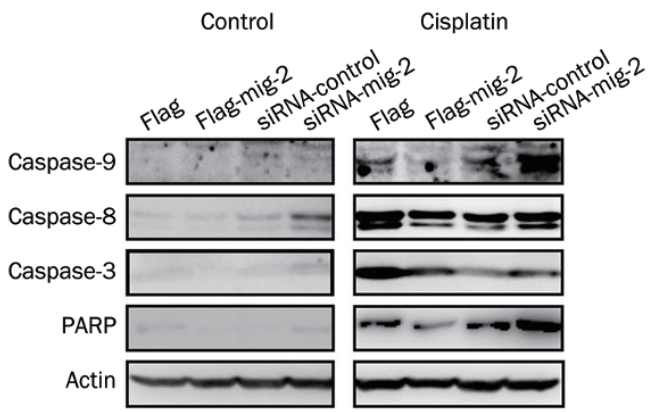

C

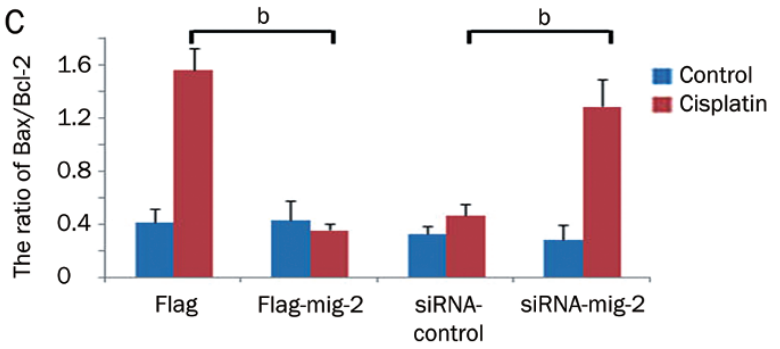

$\mathrm{E}$

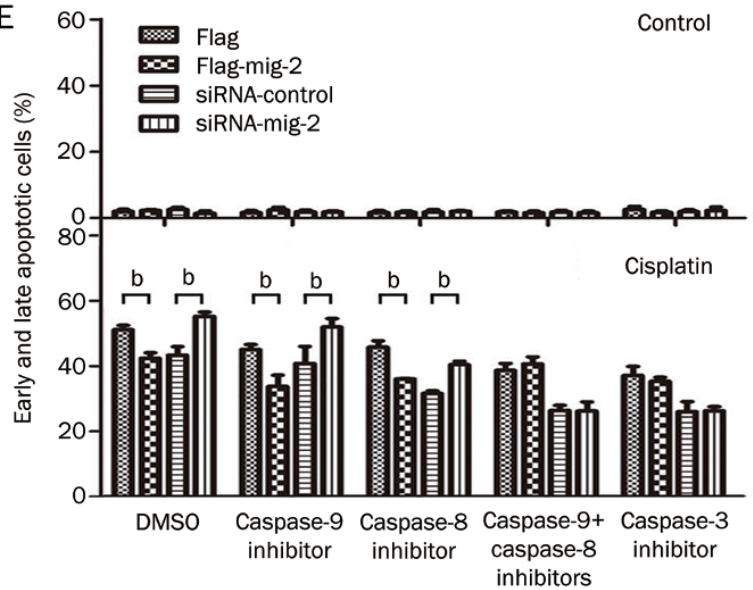

B

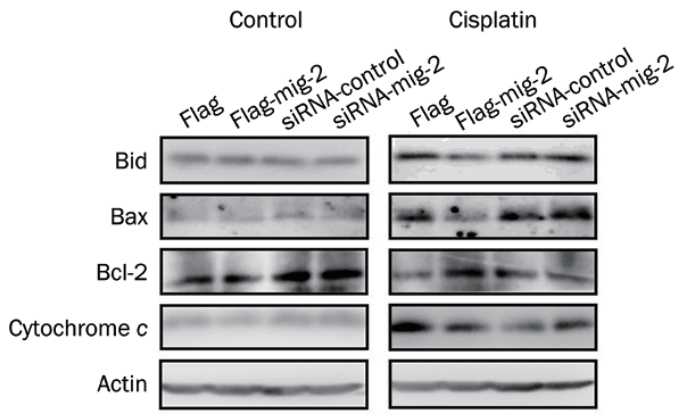

D
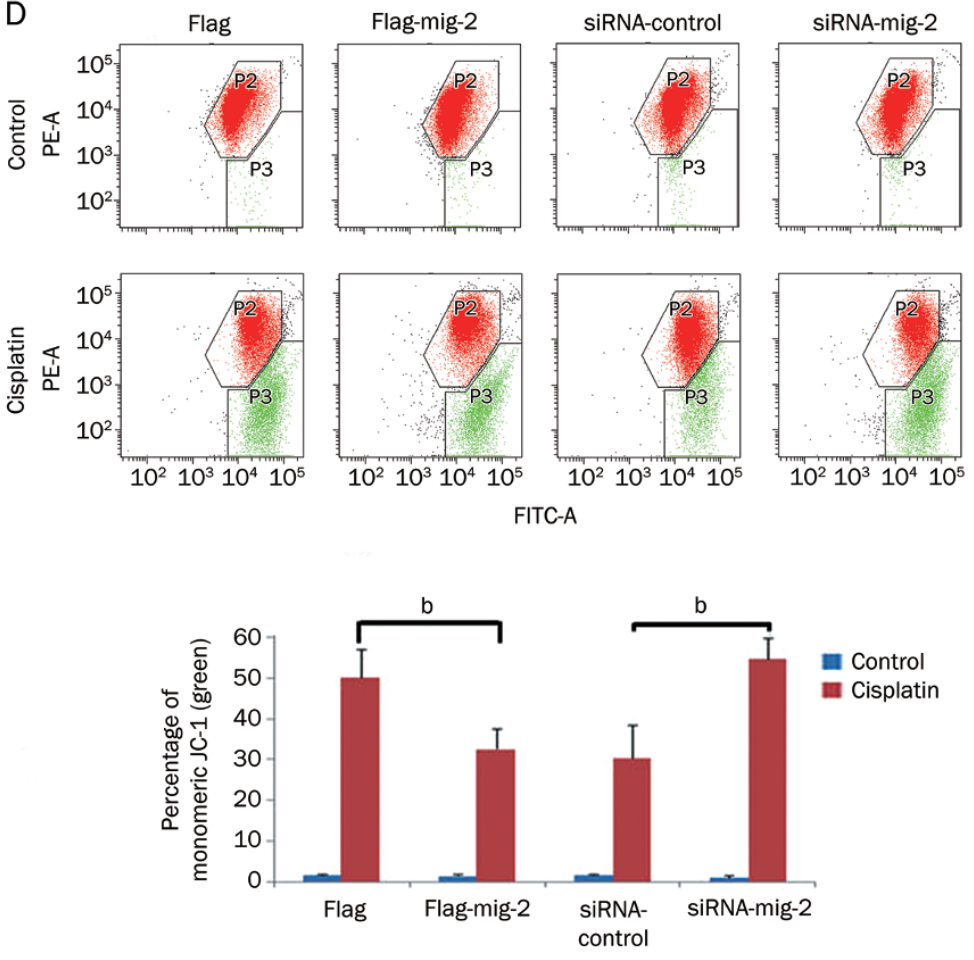

Figure 2. Mig-2-mediated cisplatin-induced apoptosis was caspase-dependent in $\mathrm{H} 4$ cells. Ectopic expression or knock-down of mig-2 in $\mathrm{H} 4$ cells treated with $40 \mu \mathrm{mol} / \mathrm{L}$ of cisplatin for $24 \mathrm{~h}$. (A and B) Western blotting analysis of cleaved-PARP, cleaved-caspase-3, cleaved-caspase-9, cleavedcaspase-8, activation of Bid, Bax, Bcl-2 and cytochrome c. (C) The ratio of Bax/Bcl-2. (D) Top: The mitochondrial membrane potential was determined by JC-1 staining. Red: aggregate JC-1; Green: monomeric JC-1. Bottom: The bar chart shows the percentage of monomeric JC-1. (E) Analysis of mig2-mediated cisplatin-induced apoptosis when cells were pretreated with caspase-9 inhibitor (Z-LEHD-FMK), caspase-8 inhibitor (Z-IETD-FMK), the combined caspase- 9 plus caspase- 8 inhibitors or caspase- 3 inhibitor (Z-DEVD-FMK) for $1 \mathrm{~h}$. The data are presented as the mean \pm SD from three independent experiments. ${ }^{\mathrm{b}} \mathrm{P}<0.05$ versus control. 
enous mig-2 expression sharply increased the cleavage of Bid expression and cytochrome $c$ release, while Bax was elevated and $\mathrm{Bcl}-2$ was diminished, which contributed to increasing the ratio of Bax/Bcl-2 (Figure 2B, 2C). Taken together, these results indicate that the mitochondrial pathway is involved in mig-2-mediated cisplatin-induced apoptosis. To confirm the involvement of mitochondria in the induction of apoptosis by mig-2, we investigated mitochondrial depolarization by JC-1 probing in cells treated or untreated with cisplatin. Compared with the control group, ectopic overexpression of mig-2 significantly repressed $\Delta \psi \mathrm{m}$, and knock-down of mig-2 drastically increased $\Delta \psi \mathrm{m}$ in cisplatin-induced apoptosis.

Caspase- 8 and caspase- 9 are initiator caspases in the extrinsic and intrinsic (mitochondrial) apoptotic pathways, respectively. Active caspase- 8 can directly activate downstream caspase-3 to induce the extrinsic apoptotic pathway, and it can activate Bid to trigger the intrinsic apoptotic pathway ${ }^{[10]}$. Therefore, Bid links the extrinsic and intrinsic apoptotic pathways $^{[11]}$. To further examine which pathways were required for mig-2-mediated cisplatin-induced apoptosis, cells were pretreated with Z-LEHD-FMK9 (caspase-9 inhibitor), Z-IETDFMK (caspase-8 inhibitor), or Z-DEVD-FMK (caspase-3 inhibitor) (final concentration $25 \mu \mathrm{mol} / \mathrm{L}$ ) for $1 \mathrm{~h}$, and then treated with cisplatin. As shown in Figure 2E, caspase-9 and caspase- 8 inhibitors could not alone affect the mediation of mig-2 in cisplatin-induced apoptosis, while pretreatment with caspase- 3 inhibitor or the combined caspase- 9 plus caspase- 8 inhibitors did not block mig-2 cell apoptosis triggered by cisplatin in $\mathrm{H} 4$ cells.
Mig-2 antagonizes cisplatin-induced apoptosis through AKT/JNK and $\mathrm{AKT} / \mathrm{p} 38$ signaling pathways

The mitogen-activated protein kinase (MAPK) signaling pathway plays an important role in chemotherapeutic druginduced apoptosis ${ }^{[12]}$. Therefore, we next examined whether MAPK signaling was activated in mig-2-mediated cisplatininduced apoptosis. Intriguingly, after treatment with cisplatin, although mig-2 could not affect the total expression of JNK and p38, it negatively regulated JNK and p38 activation (phosphorylated forms), while mig-2 had little influence on the total expression and activation of ERK1/2 and ERK5. We also investigated alterations of AKT, which is the upstream regulator of the JNK and p38 pathways, and found that mig-2 positively regulated the activation of AKT when cells were treated with cisplatin, while mig-2 did not affect the total expression of AKT (Figure 3A). To further clarify whether JNK, p38, and AKT activation play dominant roles in mig2-mediated cisplatin-induced apoptosis, we incubated cells with SP600125 (JNK inhibitor), SB203580 (p38 inhibitor), and LY294002 (AKT inhibitor) (final concentration $25 \mu \mathrm{mol} / \mathrm{L}$ ) for $1 \mathrm{~h}$ before treatment with cisplatin. When pretreated with SP600125 or SB203580 alone, mig-2 significantly attenuated cisplatin-induced apoptosis. However, the combined pretreatment with SP600125 plus SB203580 efficiently reversed mig-2 function in cisplatin-induced apoptosis; similar results were observed in LY294002-treated cells (Figure 3B). These results suggest that mig-2 antagonizes cisplatin-induced apoptosis through the AKT/JNK and AKT/p38 signaling pathways.
A

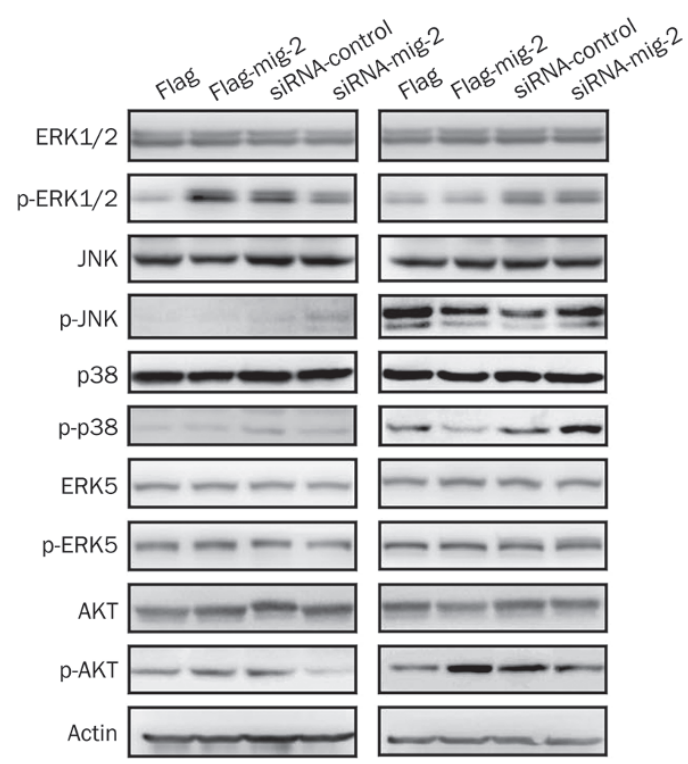

B

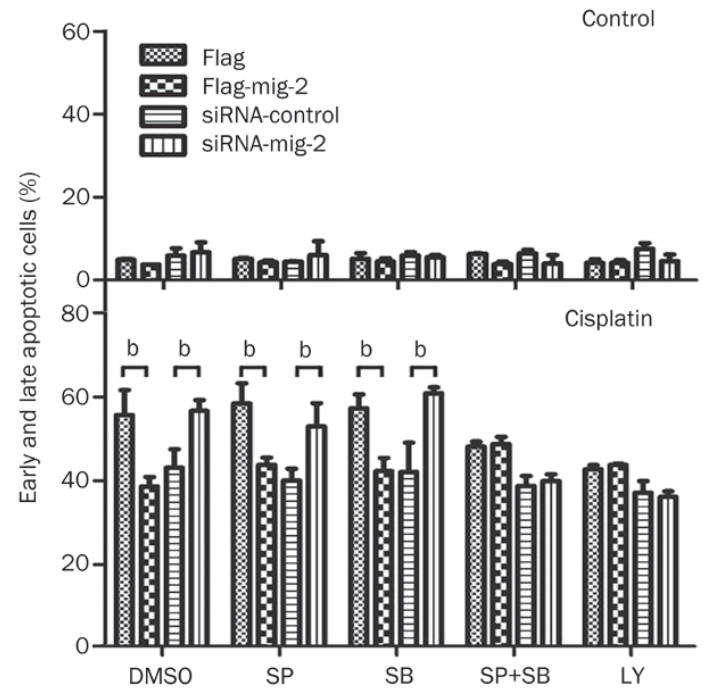

Figure 3. Analysis of pathways involved in mig-2-mediated cisplatin-induced apoptosis in $\mathrm{H} 4$ cells. Ectopic expression or knock-down of mig-2 in $\mathrm{H} 4$ cells treated with $40 \mu \mathrm{mol} / \mathrm{L}$ of cisplatin for $24 \mathrm{~h}$. (A) Western blotting analysis of the total expression and activation of ERK1/2, JNK, p38, ERK5 and AKT. (B) Analysis of mig-2-mediated cisplatin-induced apoptosis when cells were pretreated with JNK inhibitor (SP600125, SP), p38 inhibitor (SB203580, $\mathrm{SB}$ ) or AKT inhibitor (LY294002, LY) for $1 \mathrm{~h}$. The data are presented as the mean \pm SD from three independent experiments. ${ }^{b} P<0.05$ versus control. 
F3 subdomain of mig-2 is necessary and sufficient for mig-2 to antagonize cisplatin-induced apoptosis

To facilitate structure-function analysis, we attempted to identify which domain of mig-2 is required for mig-2-mediated apoptosis. GFP-tagged mig-2 mutant plasmids were constructed. GFP-mig-2 (1-541) lacked the F3 subdomain and GFP-mig-2 F3 contained only the F3 subdomain. The mig-2 wild-type and mutant plasmids were transiently transfected in $\mathrm{H} 4$ cells and their expression levels were confirmed using Western blotting (Figure 4A). Annexin V/PI staining was performed to analyze cisplatin-induced apoptosis. GFPmig-2 F3, similar to GFP-mig-2, antagonized cisplatin-induced apoptosis, while GFP-mig-2 (1-541) did not efficiently inhibit cisplatin-induced apoptosis (Figure 4B). In addition, similar to the mig-2 wild-type vector, the GFP-mig-2 F3 plasmid had the same efficiency in mediating the activation of caspase-8, caspase-9, caspase-3, Bid, JNK, p38, and AKT, and the expression of cleaved-PARP, Bcl-2, Bax, and cytochrome $c$ in cisplatininduced apoptosis (Figure $4 \mathrm{C}$ ). These results indicate that the F3 subdomain, but not other regions, is required for mig2-mediated cisplatin-induced apoptosis.

A

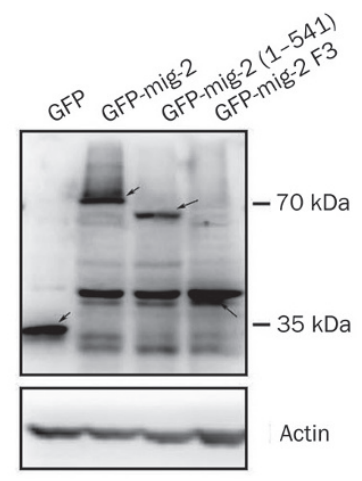

B

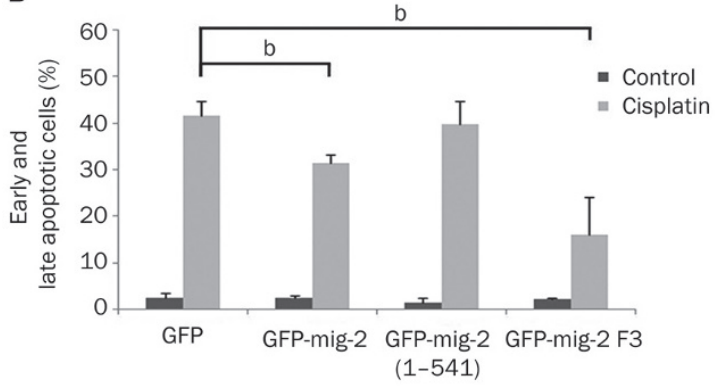

\section{Discussion}

In this study, we observed that mig-2 was expressed in glioma cells and played an important role in protecting glioma cells from cisplatin-induced apoptosis. At the molecular level, we showed that mig- 2 can repress extrinsic and intrinsic apoptotic pathways to antagonize cisplatin-induced apoptosis in glioma cells. Furthermore, mig-2 attenuated cisplatin-induced apoptosis through the AKT/JNK and AKT/p38 signaling pathways. Finally, we determined that the F3 subdomain of mig-2 was necessary and sufficient for mig-2 antagonism of cisplatininduced apoptosis in glioma cells.

Several apoptotic pathways are involved in programmed cell death. Two major caspase-dependent pathways can be classified as follows: the caspase-9-mediated intrinsic (mitochondrial) pathway and the caspase-8-mediated extrinsic pathway $^{[13]}$. In the mitochondrial pathway, the crucial step is cytochrome $c$ release. Bcl-2 family members, which consist of pro-apoptotic (such as Bax, Bak, and Bid) and antiapoptotic (such as Bcl-2, Bcl-xL, and $\mathrm{Bcl}-\mathrm{w}$ ) proteins, play a key role in mediating cytochrome $c$ release ${ }^{[14]}$. Bcl-2 and Bcl$\mathrm{xL}$ can change the inner mitochondrial permeability transition

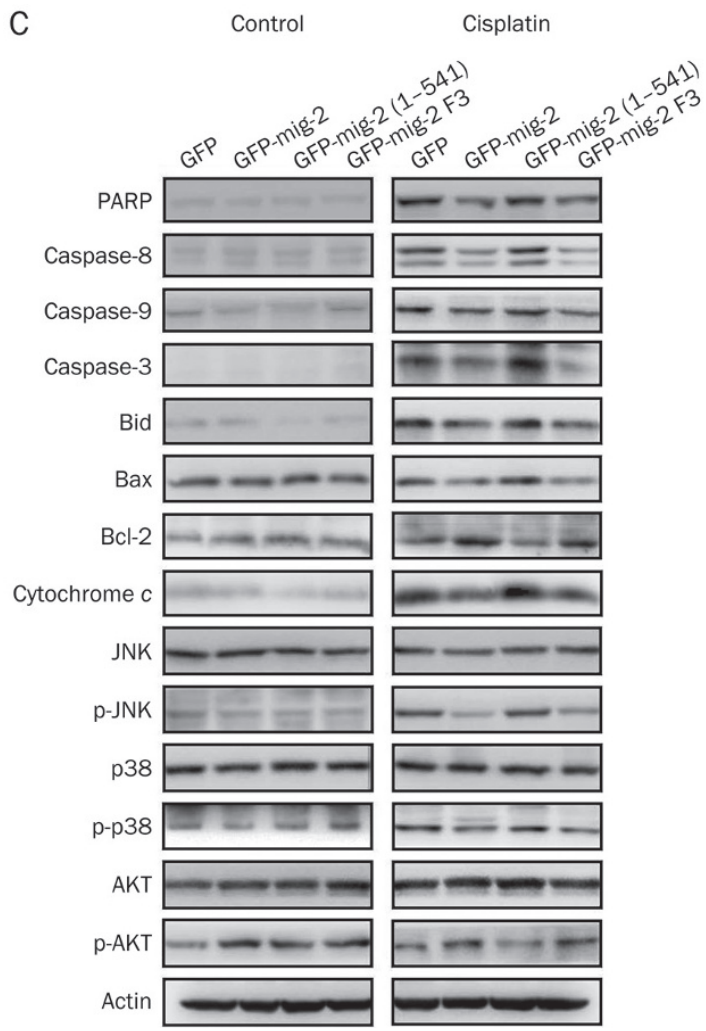

Figure 4. F3 subdomain of mig-2 is necessary and sufficient for mig-2 antagonizing cisplatin-induced apoptosis. (A) The ectopic expression of mutant and wild-type mig-2 in the glioma cells line $\mathrm{H} 4$ was confirmed by Western blotting using an anti-GFP antibody (arrows). (B) Analysis of cisplatin-induced apoptosis in mutants or wild-type mig-2 cells using Annexin V/PI staining. The bar chart shows the percentage of apoptotic cells (early apoptotic+late apoptotic). (C) Western blotting analysis of cleaved-PARP, cleaved-caspase-8, cleaved-caspase-9, cleaved-caspase-3, activation of Bid, Bax, Bcl-2, and cytochrome $c$. The total expression and activation of JNK, p38, and AKT after cells were treated or not treated with cisplatin for $24 \mathrm{~h}$ in mutant or wildtype mig- 2 cells. The data are presented as the mean \pm SD from three independent experiments. ${ }^{b} P<0.05$ versus control. 
pore and repress dissipation of mitochondrial transmembrane potential to block cytochrome $c$ release, while Bax promotes the reduction of the mitochondrial transmembrane potential to induce cytochrome $c$ release. After release to the cytosol, cytochrome $c$ facilitates the recruitment and activation of caspase-9, which subsequently triggers caspase-3 activation ${ }^{[15]}$. In the extrinsic pathway, the initiators of extracellular death receptor form a death-inducing signaling complex through recruitment of the Fas-associated death domain protein and pro-caspase- 8 , in turn activating caspase- 8 , which can directly activate downstream caspase- $3^{[16]}$. However, in some cells, the two pathways are linked by Bid, which can be activated by activation of caspase- 8 and subsequent activation of Bax and Bak to trigger the mitochondrial apoptotic pathway ${ }^{[11]}$. In this study, we found that mig-2 antagonized cisplatin-induced apoptosis through caspase-dependent pathways in glioma cells. During cisplatin-induced apoptosis, mig-2 negatively regulated the activation of caspase- 8 , caspase- 9 , and caspase- 3 , implying that mig-2 may mediate extrinsic and/or intrinsic pathways to protect glioma cells from cisplatin. Our investigations revealed that mig-2 can up-regulate Bcl-2, inhibit Bax expression, induce mitochondrial transmembrane potential loss and release cytochrome $c$, suggesting that the mitochondrial pathway is involved in mig-2-mediated cisplatin-induced apoptosis. We also observed that the cleaved form of Bid was negatively regulated by mig-2, indicating that activation of caspase- 8 can activate Bid to participate in the mitochondrial pathway in mig-2-mediated apoptosis. Finally, when pretreated by the inhibition of caspase- 8 or caspase- 9 alone, mig- 2 also can protect glioma cells from cisplatin, but mig-2 can not affect cisplatin-induced apoptosis in glioma cells when pretreated with the combined caspase- 9 plus caspase- 8 inhibitors or caspase- 3 inhibitor. Therefore, mig-2 may act via extrinsic and intrinsic pathways to inhibit cisplatin-induced apoptosis.

The MAPK pathway, which can be activated by several stimuli and plays important roles in cell proliferation, apoptosis and differentiation ${ }^{[17]}$, contains three major subfamilies harboring the ERK1/2, JNK, and p38 pathways. However, it was reported recently that a fourth subfamily (ERK5 pathway) also has a critical function in the mediation of apoptosis ${ }^{[18]}$. Therefore, we examined the total and phosphorylated expression levels of the MAPK family members ERK1/2, JNK, p38, and ERK5 in mig-2-mediated cisplatin-induced apoptosis. The results showed that mig- 2 negatively regulated the phosphorylated forms of JNK and p38, but did not affect the activation of ERK1/2 and ERK5 in cisplatin-induced apoptosis. In addition to enhancing the activation of caspase-8, the JNK and p38 pathways are also sufficient to activate Bax, as well as the release of cytochrome $c$ and the onset of mitochondrial apoptosis $^{[19]}$. These results suggest that the JNK and p38 pathways are involved in mig-2-mediated cisplatin-induced apoptosis in glioma cells. The AKT pathway is critical for regulating cell survival and blocking apoptosis, and acts as a hub in regulating multi-pathways, such as the AKT/mTOR, AKT/NF-kB and AKT/GSK3 $\beta$ pathways ${ }^{[20]}$. AKT also can repress JNK and p38 pathways to inhibit apoptosis. AKT represses apoptosis signal-regulating kinase 1 (ASK1) activation by directly phosphorylating Ser83. Inactivation of ASK1 would not activate mitogen-activated protein kinase kinase 4 (MKK4), MKK7, MKK3, or MKK6 for activating JNK and p38 pathways ${ }^{[21]}$. In our study, we found that mig-2 could positively meditate AKT activation in cisplatin-induced apoptosis. Therefore, to further investigate which pathways were required for mig-2-mediated apoptosis, we blocked JNK, p38, and AKT pathways using inhibitors. When pretreated with JNK or p38 inhibitors alone, mig-2 significantly antagonized cisplatin-induced apoptosis. However, pretreatment with combined JNK and p38 inhibitors exhibited a similar effect to that of the AKT inhibitor in blocking the protective function of mig-2 in cisplatin-induced apoptosis, indicating that mig-2 at least partly attenuates cisplatininduced apoptosis through AKT/JNK and AKT/p38 signaling pathways in glioma cells.

Mig-2, which belongs to the kindlin family, plays key roles in regulating cell-ECM adhesion and migration ${ }^{[4]}$. Although mig-2 does not contain catalytic domains, it has multiple molecular interaction domains, including an N-terminal F0 domain and C-terminal FERM domains, which comprise F1, F2, and F3 subdomains. The F2 subdomain is divided by a $\mathrm{PH}$ domain. Together with the N-terminal domain, the F3 subdomain mediates integrin activation by interacting with integrin $^{[3]}$. The N-terminal domain also can bind with migfilin or phosphatidylinositol-(4,5)-bisphosphate (PIP2) to mediate cell motility, cell shape or anchor mig-2 onto the membrane, which is also attributed to $\mathrm{PH}$ domain interaction with phosphatidylinositol-(3,4,5)-trisphosphate (PIP3) ${ }^{[4,22]}$. Gong et al reported that mig-2 protects prostate cancer cells from cisplatin-induced apoptosis ${ }^{[7]}$; however, which domain is required for mig-2-mediated apoptosis remains unclear. Here, we demonstrated that the F3 subdomain was necessary and sufficient for mig-2 inhibition of cisplatin-induced apoptosis in glioma cells, and the underlying mechanisms were also dependent on F3 subdomain involvement.

In conclusion, we demonstrate the function and underlying mechanism of mig-2 in antagonizing cisplatin-induced apoptosis in glioma cells. In addition to better understanding the mechanisms of apoptosis in glioma cells, our findings provide an important theoretical background for clinical applications of mig-2, basing individual therapy according to the expression of mig-2 in combination with platinum-based chemotherapy for patients with glioma. Additionally, the combination of chemotherapeutic drugs with mig-2 blockade may efficiently improve the curative effect in patients with glioma. The expression level of mig-2 may also be a predictive biomarker for drug resistance in glioma.

\section{Acknowledgements}

This work is supported by the 973 National Key Fundamental Research Program of China (2012CB967003 and 2011CB910801) and the National Natural Science Foundation of China (81071633). 


\section{Author contribution}

Yun-wei OU designed the project and wrote the manuscript; Zi-tong ZHAO performed some experiments; Chuan-yue WU, Bai-nan XU, Yong-mei SONG, and Qi-min ZHAN provided supervision and support in the project.

\section{References}

1 Johannesen TB, Langmark F, Lote K. Cause of death and long-term survival in patients with neuro-epithelial brain tumours: a populationbased study. Eur J Cancer 2003; 39: 2355-63.

2 Liu WT, Huang CY, Lu IC, Gean PW. Inhibition of glioma growth by minocycline is mediated through endoplasmic reticulum stressinduced apoptosis and autophagic cell death. Neuro Oncol 2013; 15 : $1127-41$.

3 Ma YQ, Qin J, Wu C, Plow EF. Kindlin-2 (Mig-2): a co-activator of beta3 integrins. J Cell Biol 2008; 181: 439-46.

4 Tu Y, Wu S, Shi X, Chen K, Wu C. Migfilin and Mig-2 link focal adhesions to filamin and the actin cytoskeleton and function in cell shape modulation. Cell 2003; 113: 37-47.

5 Zhao T, Guan L, Yu Y, Pei X, Zhan J, Han L, et al. Kindlin-2 promotes genome instability in breast cancer cells. Cancer Lett 2013; 330: 208-16.

6 Shen Z, Ye Y, Kauttu T, Seppanen H, Vainionpaa S, Wang S, et al. Novel focal adhesion protein kindlin-2 promotes the invasion of gastric cancer cells through phosphorylation of integrin beta1 and beta3. J Surg Oncol 2013; 108: 106-12.

7 Gong X, An Z, Wang Y, Guan L, Fang W, Stromblad S, et al. Kindlin-2 controls sensitivity of prostate cancer cells to cisplatin-induced cell death. Cancer Lett 2010; 299: 54-62.

8 Ou Y, Ma L, Dong L, Zhao Z, Zhou W, Fan J, et al. Migfilin protein promotes migration and invasion in human glioma through epidermal growth factor receptor-mediated phospholipase C-gamma and STAT3 protein signaling pathways. J Biol Chem 2012; 287: 32394-405.

9 Fan J, Ou YW, Wu CY, Yu CJ, Song YM, Zhan QM. Migfilin sensitizes cisplatin-induced apoptosis in human glioma cells in vitro. Acta Pharmacol Sin 2012; 33: 1301-10.

10 Jiang X, Wang X. Cytochrome c-mediated apoptosis. Annu Rev
Biochem 2004; 73: 87-106.

11 Almasan A, Ashkenazi A. Apo2L/TRAIL: apoptosis signaling, biology, and potential for cancer therapy. Cytokine Growth Factor Rev 2003; 14: $337-48$.

12 Hsiao PC, Hsieh YH, Chow JM, Yang SF, Hsiao M, Hua KT, et al. Hispolon induces apoptosis through JNK1/2-mediated activation of a caspase-8, -9, and -3-dependent pathway in acute myeloid leukemia (AML) cells and inhibits AML xenograft tumor growth in vivo. J Agric Food Chem 2013; 61: 10063-73.

13 Ou Y, Ma L, Huang Z, Zhou W, Zhao C, Zhang B, et al. Overexpression of cyclin B1 antagonizes chemotherapeutic-induced apoptosis through PTEN/Akt pathway in human esophageal squamous cell carcinoma cells. Cancer Biol Ther 2013; 14: 45-55.

14 Eskes R, Desagher S, Antonsson B, Martinou JC. Bid induces the oligomerization and insertion of Bax into the outer mitochondrial membrane. Mol Cell Biol 2000; 20: 929-35.

$15 \mathrm{Kim}$ R, Emi M, Tanabe K. Role of mitochondria as the gardens of cell death. Cancer Chemother Pharmacol 2006; 57: 545-53.

16 Ghobrial IM, Witzig TE, Adjei AA. Targeting apoptosis pathways in cancer therapy. CA Cancer J Clin 2005; 55: 178-94.

17 Chan-Hui PY, Weaver R. Human mitogen-activated protein kinase kinase kinase mediates the stress-induced activation of mitogenactivated protein kinase cascades. Biochem J 1998; 336: 599-609.

18 Tian W, Zhang Z, Cohen DM. MAPK signaling and the kidney. Am J Physiol Renal Physiol 2000; 279: F593-604.

19 Wu CM, Chen ZW, Chen TH, Liao JW, Lin CC, Chien MS, et al. Mitogenactivated protein kinases p38 and JNK mediate Actinobacillus pleuropneumoniae exotoxin Apxl-induced apoptosis in porcine alveolar macrophages. Vet Microbiol 2011; 151: 372-8.

20 Hemmings BA. Akt signaling: linking membrane events to life and death decisions. Science 1997; 275: 628-30.

21 Aikin R, Maysinger D, Rosenberg L. Cross-talk between phosphatidylinositol 3-kinase/AKT and c-jun $\mathrm{NH}_{2}$-terminal kinase mediates survival of isolated human islets. Endocrinology 2004; 145: 4522-31.

22 Liu Y, Zhu Y, Ye S, Zhang R. Crystal structure of kindlin-2 PH domain reveals a conformational transition for its membrane anchoring and regulation of integrin activation. Protein Cell 2012; 3: 434-40. 